\title{
EVOLUTION FROM THE AGB: VARIABILITY
}

\author{
DIMITAR D. SASSELOV \\ Harvard-Smithsonian Center for Astrophysics, Cambridge, MA 02138, USA
}

\begin{abstract}
Luminous low-mass stars of intermediate $T_{\text {eff }}$ - candidates for post-AGB transition objects to PNNi, are all expected to be pulsationally variable. Different modes and their combinations may be involved, leading to a variety of observed behaviour.
\end{abstract}

\section{Introduction}

All stars on and close to the asymptotic giants branch (AGB) are intrinsic variable stars. This variability involves dynamical time scales (months) and is generally caused by large scale motions of the stellar envelope, which will be referred to as pulsations for simplicity.

The pulsations of a stellar envelope can (and appear to) influence strongly the stellar AGB and post-AGB evolution. Such pulsations cannot influence the nuclear burning regions of a star, yet they affect most of the volume of its interior - the extended, non-adiabatic, envelope. Thus pulsation is related to or, in fact, drives mass loss and may determine the onset and scale of post-AGB evolution (Schönberner \& Blöcker 1992; Wood, this volume).

Intrinsic stellar variability is also a very powerful diagnostic tool for stellar interiors and evolution. This is especially true for post-AGB stars which evolve on comparatively very short time scales.

\section{Classes of Variable Stars}

The region of the HR diagram of the AGB and to immediately hotter $T_{\text {eff }}$ is populated with a large variety of different variable stars. However, their differences are often superficial or misleading, because many classes of variable stars defined earlier in this century were based mainly on light curves criteria alone.

There are four classes of variables that are relevant to our discussion of post-AGB objects: RV Tauri, UU Herculis, SRd, and R CrB stars. The variability of central stars of planetary nebulae is outside the scope of our discussion.

\subsection{RV Tauri stars}

This is a small class of pulsating variable stars, apparently luminous, and with a characteristic light curve - alternating deep and shallow minima. A handful of stars with similar light curves are known in globular clusters; most field members have peculiar infrared excesses (Jura 1986); several exhibit decreasing pulsation periods (Percy et al. 1991), and as 
a consequence the RV Tauri stars are usually associated with low-mass post-AGB stellar variability. It should be remembered however, that the RV Tauri phenomenon refers only to brightness variability in $\mathrm{F}-\mathrm{K}$ giants, while the RV Tauri light-curve behaviour has not been proven to arise in low-mass stars alone (see review by Wahlgren 1992).

There are no known RV Tau stars in binaries. There are no established Magellanic Clouds members either. As a result their luminosities are poorly known.

\subsection{UU Herculis stars}

This is an even smaller class of pulsating variable stars, apparently luminous, at high galactic latitudes, and low-amplitude Cepheid-like (but unstable) light curves. Their variability, though peculiar (Fernie 1992), is not a straightforward discriminant. They are of class $\mathrm{F}$ to early $\mathrm{G}$, and span in metalicity from deficient to solar. Many of them were found to have substantial IR excess and were associated with transit objects between the AGB and PNNi. Despite the largely disparate characteristics of these high-latitude objects, it is noteworthy that there are much more (known and suspected) spectroscopic binaries among the UU Her stars, than for any of the other three classes here (Waelkens, 1992). However, parameters for any of these binaries are yet to be determined. No Magellanic Clouds members are known.

\section{3. $R$ CrB stars}

The $\mathrm{R}$ CrB stars have strong features as a class (occasional deep minima), and seem less prone to selection effects. However, their semi-regular pulsational variability falls within the general definition of the SRd class. The $\mathrm{R} \mathrm{CrB}$ stars are few; none are known in binaries, and just a couple are found in the Magellanic Cloud. They occupy the same region of the HR diagram as the above classes, but their evolutionary status is not clear (see Whitney, Soker, and Clayton 1991).

\subsection{SRd stars}

The yellow semi-regular stars (SRd) class has a very loose definition: it contains almost all yellow giants and supergiants which are neither Cepheids nor RV Tauri stars. This is a disparate mixture of Population I and II stars, as well as other sparsely observed variables (like RV Tauri or UU Herculis) hastily assigned to it.

In addition to the classified stars, there are also others that are loosely associated with some of the above classes and our topic, like FG Sge and $\epsilon$ Aur (F).

\section{Models}

On the HR diagram these four classes of variable stars and the post-AGB transition objects, are expected to coexist with evolved massive Population I stars. Most notable of these - the classical Cepheids, define a narrow instability strip in that region. Similar temperatures and luminosities, but different masses, imply a very different internal structure, hence pulsation characteristics.

A comparison between a low-mass post-AGB star at spectral class F, and a Cepheid of the same $T_{\text {eff }}$ and $L$ (but 10 times more massive) is instructive. On one hand both stars have very similar structure with respect to a radial mass coordinate. Hence, the driving mechanisms of their oscillations are similar. On the other hand, the two stars are very different with respect to a radial depth coordinate: the low-mass star contains most of its 
mass in the tiny hot core (of less than $0.01 R_{\mathrm{ph}}$ ), which is surrounded by an extended envelope with virtually zero density gradient. As a result, any pulsations excited in such a low-mass supergiant are going to be strongly non-adiabatic and non-linear. For example, a $0.8 M_{\odot}, 20000 L_{\odot}$ star with $T_{\text {eff }}=5500 \mathrm{~K}$ will have a thermal time-scale $t_{\text {th }} \approx 4$ days in its envelope. However, the envelope dynamical time-scale will be much greater, $t_{\mathrm{dyn}} \approx 40$ days, leading to strong non-linear effects (see theoretical models by Fadeyev (1984), Saitou, Takeuti, and Tanaka (1989), Aikawa (1991) and others).

One of the most reliable facts established by the different theoretical models to-date, is that luminous low-mass stars of intermediate $T_{\text {eff }}$ are unstable to different modes of pulsation through a wide range of temperatures and luminosities. This is in contrast to the massive stars in that region of the HR diagram, in which pulsation is strongly confined in parameter space. In the luminous low-mass stars radial modes different from the fundamental are often dominant, and this may explain the observed preference towards lower amplitudes of pulsation (see Aikawa 1992). As can be expected, the pulsational behaviour of luminous low-mass stars is very unstable and complex.

In general the current theoretical understanding of the pulsations of luminous low-mass stars remains very poor. One important challenge is the treatment of convection (Saitou 1992). The effects of radiative transfer have been shown to be significant, as well (Zalewski 1992).

So, we might expect a post-AGB transition object to look like an intermediate temperature supergiant, pulsating with a period of about 1-2 months, and more often with low amplitude (below $0.2 \mathrm{mag}$ ), and almost certainly in a semi-regular fashion. While no specific form of the light curve can be ascribed to the post-AGB objects, there is no good understanding of the peculiar light curves observed among likely candidates (Zsoldos 1990; Fernie 1992). The models point to the essential similarity between SRd and RV Tau light curves (Takeuti 1992; Aikawa 1992). However, such results are more strongly affected by the problems mentioned above, as well as by the treatment of the stellar atmosphere, and should be used with caution.

\section{Pulsation and Evolution}

The evolution of stars from the AGB to the white dwarf phase (towards higher temperatures and smaller radii) is very fast by normal stellar standards. A temperature increase at a mean rate of several Kelvins per year is expected (Schönberner \& Blöcker 1992). Such a rapid increase should lead to detectable color and period changes on a time scale of decades. No completely convincing example has been observed yet, but there are several very hopeful cases.

Potentially, the pulsation period of a star is a more sensitive indicator of evolutionary change than is color. This is especially true for classes of variables, like RV Tauri and UU Herculis, with stable periods (despite light curve instabilities). Generally, the pulsation period, $\mathrm{P} \sim T_{\text {eff }}^{-n}$, where $n$ has values between 3.0 and 3.7, depending on the model's density distribution and the mode involved. The temperature, $T_{\text {eff }}$, increases with time at a constant luminosity, $L$, as determined by the evolutionary calculations, e.g. Schönberner \& Blöcker (1992). The stellar luminosity, $L$, is a parameter. Thus obtained, the rate of change of the pulsation period is a robust result of general validity: for radial or non-radial, non-adiabatic, and non-linear oscillations (see Bruggen and Smeyers 1987). 
The expected rates of period change are between 0.1 and 2 days per decade; the pulsation period decreases. Such period changes are indeed observed in five RV Tauri stars with extensive photometric data, which may confirm that at least some RV Tauri stars are postAGB objects.

\section{Conclusions}

Stellar pulsation on the AGB and after the AGB is intimately related to the driving, the amount, and the spacial distribution of mass loss. Thus pulsation may determine the speed of post-AGB evolution, as well as play a role in shaping the planetary nebula (e.g. the inner axisymmetric regions of NGC 6826 and NGC 6543, see Soker and Harpaz 1992).

Stellar pulsation on the AGB and after the AGB is the rule, rather than the exception. This is a theoretical expectation for single stars, but is also true for binaries. The probability that most AGB and post-AGB stars have companions, often in a common envelope, is very high (see Livio, this volume). The stellar oscillations excited even by a Jupiter-like companion may be significant and cause low-amplitude semi-regular variability (Soker 1992). This adds additional variety to the types of oscillation modes that can be exhibited by postAGB objects and suggests more caution when using traditional variable star classes. Light curve characteristics may not have a direct relation to evolutionary states, as exemplified by RV Tauri and SRd stars, as well as SRa and SRb stars (see Kerschbaum and Hron, this volume).

\section{References}

Aikawa, T. 1991, ApJ, 374, 700.

Aikawa, T. 1992, MNRAS, in press.

Bruggen, P., and Smeyers, P. 1987, A\&A, 186, 170.

Fadeyev, Yu.A. 1984, Ap\&SS, 100, 329.

Fernie, J. D. 1992, in Luminous High-Latitude Stars, ed. D. Sasselov, ASP Conf. Series, in press.

Jura M. 1986, ApJ, 309, 732.

Percy, J.R., Sasselov, D.D., Alfred, A., \& Scott, G. 1991, ApJ, 375, 691.

Saitou, M. 1992, in Non-Linear Phenomenae in Stellar Variability, IAU Colloq. 134, eds. M.Takeuti and J.R.Buchler, Ap\&SS, in press.

Saitou, M., Takeuti, M., and Tanaka, Y. 1989, PASJ, 41, 297.

Soker, N. 1992, ApJ, 386, 190.

Soker, N., and Harpaz, A. 1992, PASP, in press.

Schönberner, D., and Blöcker, T. 1992, in Luminous High-Latitude Stars, ed. D. Sasselov, ASP Conf. Series, in press.

Takeuti, M. 1992, ibid.

Waelkens, C. 1992, ibid.

Wahlgren, G.M. 1992, ibid.

Whitney, B.A., Soker, N., and Clayton, G. C. 1991, AJ, 102, 284.

Zalewski, J. 1992, PASJ, 44, 27.

Zsoldos E. 1990, Ap\&SS, 165, 111. 